\title{
Esthesioneuroblastoma, Neuroendocrine Carcinoma, and Sinonasal Undifferentiated Carcinoma: Differentiation in Diagnosis and Treatment
}

\author{
Shirley Y. Su ${ }^{1}$ Diana Bell ${ }^{2}$ Ehab Y. Hanna ${ }^{1}$ \\ ${ }^{1}$ Department of Head and Neck Surgery, University of Texas MD \\ Anderson Cancer Center, Houston, Texas, United States \\ 2 Department of Pathology, University of Texas MD Anderson Cancer \\ Center, Houston, Texas, United States

\begin{abstract}
Address for correspondence Ehab Y. Hanna, MD, Department of Head and Neck Surgery, University of Texas MD Anderson Cancer Center, 1515 Holcombe Blvd., Suite 1445, Houston, TX 77030-4009, United States (e-mail: EYHanna@mdanderson.org).
\end{abstract}

Int Arch Otorhinolaryngol 2014;18:S149-S156.

\begin{abstract}
Introduction Malignant sinonasal tumors comprise less than $1 \%$ of all neoplasms. A wide variety of tumors occurring primarily in this site can present with an undifferentiated or poorly differentiated morphology. Among them are esthesioneuroblastomas, sinonasal undifferentiated carcinomas, and neuroendocrine carcinomas.

Objectives We will discuss diagnostic strategies, recent advances in immunohistochemistry and molecular diagnosis, and treatment strategies.

Data Synthesis These lesions are diagnostically challenging, and up to $30 \%$ of sinonasal malignancies referred to the University of Texas MD Anderson Cancer Center

Keywords

- sinonasal malignancy

- esthesioneuroblastoma

- sinonasal undifferentiated carcinoma

- neuroendocrine carcinoma

- olfactory neuroblastoma are given a different diagnosis on review of pathology. Correct classification is vital, as these tumors are significantly different in biological behavior and response to treatment. The past decade has witnessed advances in diagnosis and therapeutic modalities leading to improvements in survival. However, the optimal treatment for esthesioneuroblastoma, sinonasal undifferentiated carcinoma, and neuroendocrine carcinoma remain debated. We discuss advances in immunohistochemistry and molecular diagnosis, diagnostic strategies, and treatment selection.

Conclusions There are significant differences in prognosis and treatment for esthesioneuroblastoma, neuroendocrine carcinoma, and sinonasal undifferentiated carcinoma. Recent advances have the potential to improve oncologic outcomes but further investigation in needed.
\end{abstract}

\section{Introduction}

The sinonasal tract is the location for a wide variety of benign and malignant tumors. Per cubic centimeter, the sinonasal tract gives rise to a greater diversity of neoplasms than any other site in the human body. The diversity is partly due to the anatomic complexity and highly varied tissues in this compact area. Malignancies of the sinonasal tract have shown dramatic improvements in survival, from $20 \%$ in the 1950 s to 60 to $80 \%$ survival as cited by the most current literature. This advancement is closely tied to the improvements in diagnosis, surgery, and adjuvant treatments.

Malignant sinonasal tract tumors comprise less than $1 \%$ of all neoplasms and $\sim 3 \%$ of the upper aerodigestive tract. Sinonasal tract malignancies most commonly affect the maxillary sinus (60\%), followed by the nasal cavity (22\%), ethmoid sinus (15\%), and frontal and sphenoid sinuses $(<3 \%)$. Sinonasal tract tumors are diverse. The majority are squamous carcinomas and their variants (55\%), followed by nonepithelial neoplasms (20\%), glandular tumors (15\%), 
undifferentiated carcinomas (7\%), and miscellaneous tumors (3\%). The spectrum of primary sinonasal undifferentiated neoplasms has expanded because new entities specific to this region or initially described in other locations have been recognized over time.

Several of the malignant tumors occurring primarily in the sinonasal tract may present with an undifferentiated or poorly differentiated morphology and are composed of small, medium, and large round or polygonal atypical cells. These lesions pose significant diagnostic difficulties for the surgical pathologist, especially with limited biopsy material. Up to $30 \%$ of sinonasal malignancies referred to our institution are given a different diagnosis on review of pathology. However, correct classification by means of histology, immunohistochemistry, or molecular biology is important for initiating an appropriate treatment strategy.

There is significant debate regarding the optimal treatment of esthesioneuroblastoma (ENB), neuroendocrine carcinoma (NEC), and sinonasal undifferentiated carcinoma (SNUC). The rarity of these tumors, difficulties in establishing a diagnosis, and heterogeneity in treatment approaches all contribute to the lack of consensus. However, the past decade has resulted in significant progress in treatment. This review emphasizes diagnostic strategies, recent advances in immunohistochemistry and molecular diagnosis, and a discussion of treatment strategies.

\section{Review of Literature and Discussion}

\section{Esthesioneuroblastoma}

ENB is a tumor restricted to the area of olfactory neuroepithelium, which arises from embryonic olfactory placodes and in adults is replaced partially by respiratory mucosa. First described by Berger and colleagues in 1924, ENB has been characterized as a rare malignant neoplasm of the sinonasal cavity that arises in the superior portion of the nasal vault. ${ }^{1,2}$ A variety of nomenclature has been used to describe this tumor (ENB, esthesioneuroepithelioma, esthesioneurocytoma, olfactory neuroblastoma, and NEC); the accepted terms at this time are esthesioneuroblastoma and olfactory neuroblastoma. Arising from the neural-epithelial olfactory mucosa, phenotypically ENB is intermediate between that of a pure neural neoplasm (e.g., neuroblastoma and paraganglioma) and a neuroendocrine epithelial tumor (e.g., carcinoid, NEC, small cell carcinoma).

The differential diagnosis is broad: ENB can be confused histologically with several other "small blue round cell tumors" of the nasal cavity and paranasal sinuses. Tumors commonly confused with ENB include SNUC, sinonasal NEC, small cell carcinoma, pituitary adenoma, melanoma, lymphoma, and rhabdomyosarcoma. ${ }^{1}$ Therefore, thorough pathologic review and ancillary studies are essential to differentiating between these tumor types and properly diagnosing ENB. ENB typically shows diffuse staining with neuron-specific enolase, synaptophysin, and chromogranin. Cytokeratins, vimentin, epithelial membrane antigen, and CD56 can all show variable reactivity. Desmin, myogenin, vimentin, and actin are negative, important markers that rule out rhabdo- myosarcoma. S-100 is variably positive, but positive cells are usually limited to the periphery of neoplastic nests, corresponding to sustentacular cells. This characteristic pattern differentiates ENB from sinonasal melanoma. FLI1 is negative, as is the Ewings Sarcoma/Friend Leukemia Integration-1 (EWS/FLI1) chimeric transcript, ruling out the rare diagnosis of peripheral neuroectodermal tumor/Ewing sarcoma. Hyams grading scheme, which covered work predating the first description of SNUC by nearly 10 years, captures the spectrum of ENB maturation. ${ }^{1,2}$ Several groups have asserted that grade 3 and predominantly grade 4 ENBs are in fact SNUCs. ${ }^{1}$ Low-grade ENB forms sharply demarcated lobules of cells embedded in a richly vascularized stroma; the cells are small (slightly larger than lymphocytes), with "salt-and-pepper" chromatin. Neuropil (interdigitating neuronal processes) is abundant in low-grade ENB (-Fig. 1A). The cells may form rosettes with true lumens (Flexner-Wintersteiner type) or pseudorosettes (Homer-Wright type). High-grade ENBs are more difficult to diagnose, with increased cytologic atypia, pleomorphism, necrosis, increased mitotic activity, solid pattern, loss of neuropil, and abundant mitotic activity (-Fig. 1B). We retrospectively analyzed the records of 124 patients with ENB who had been treated at our institution for the association of grade and stage with prognostic outcome. High-grade ENB was significantly associated with poor outcomes, and advanced stage was not associated with poor outcome in this cohort. Grading should certainly be considered in prognostication and treatment decisions for ENB. ${ }^{3}$

Cytogenetic data for ENB are limited. Holland et al, who performed cytogenetic characterization of one case using trypsin Giemsa staining (GTG banding), multicolor fluorescence in situ hybridization, and single-nucleotide polymorphism karyotyping, reported numerous chromosomal aberrations predominantly involving chromosomes $2 \mathrm{q}, 5$, $6 \mathrm{q}, 17,19,21 \mathrm{q}$, and 22 , as well as trisomy $8 .{ }^{4}$ Bockmühl et al applied conventional comparative genomic hybridization (CGH) to $22 \mathrm{ENB}$ and reported frequent deletions of $1 \mathrm{p}, 3 \mathrm{p} / \mathrm{q}$, $9 \mathrm{p}$, and $10 \mathrm{p} / \mathrm{q}$, and amplifications of $17 \mathrm{q}, 17 \mathrm{p} 13,20 \mathrm{p}$, and 22q. ${ }^{5}$ They also noted a specific deletion on chromosome 11 and gain on chromosome $1 \mathrm{p}$, which were associated with metastasis and a worse prognosis. Three ENB were studied by Riazimand et al using conventional CGH, and amplification of whole chromosome 19; partial gains of 1p, 8q, 15q, and 22q; and deletions of $4 \mathrm{q}$ and $6 \mathrm{p}$ were detected. ${ }^{6}$ Szymas et al studied a single ENB and found gains of whole chromosomes $4,8,11$, and 14 ; partial gains of $1 \mathrm{q}$ and $17 \mathrm{q}$; partial deletions of $5 q$ and 17q; and whole chromosome losses of 16, 18, 19, and $\mathrm{X}^{7}$ Guled et al applied an oligonucleotide-based array CGH to identify DNA copy number changes in 13 cases of ENB. ${ }^{8}$ Novel chromosomal regions were identified that were frequently altered in addition to previously reported abnormal regions. The most frequent changes included gains at 7q11.22-q21.11, 9p13.3, 13q, 20p/q, and Xp/q, and losses at 2q31.1, 2q33.3, 2q37.1, 6q16.3, 6q21.33, 6q22.1, 22q11.23, 22q12.1, and Xp/q. Gains were more frequent than losses, and high-stage tumors showed more alterations than low-stage ENB. Frequent changes in high-stage tumors were gains at 13q14.2-q14.3, 13q31.1, and 20q11.21-q11.23, and loss of 


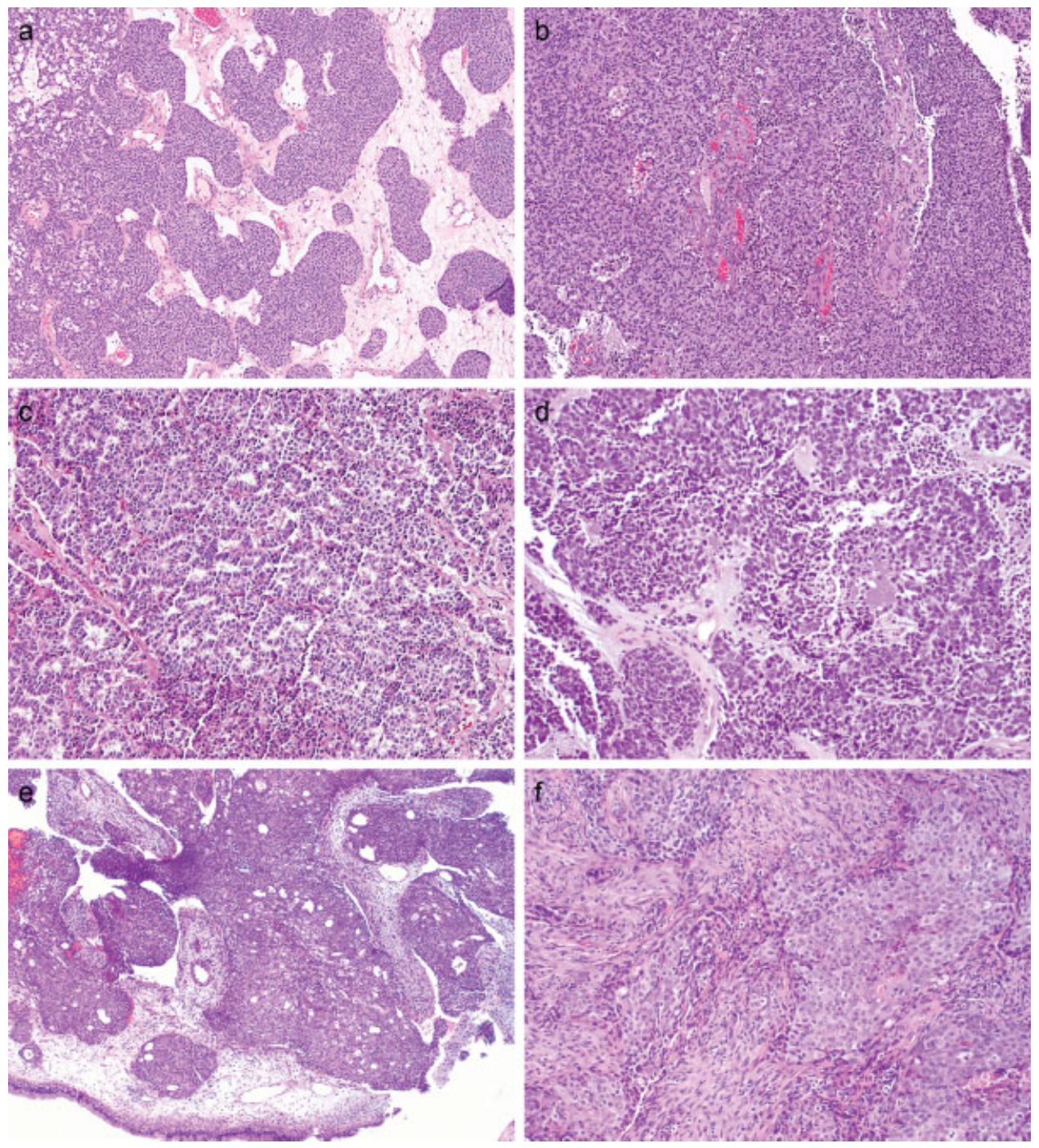

Fig. 1 (A) Low-grade esthesioneuroblastoma with lobules and sheets of monotonous-looking cells, featuring nuclei with no atypia and scant cytoplasm, abundant neurofibrillary background, and vascularized stroma. (B) High-grade esthesioneuroblastoma with solid pattern and decreased neuropils, increased cytologic atypia, and mitosis. (C) Carcinoid, with cords of uniform, bland cells with central nuclei and moderate granular cytoplasm. (D) High-grade neuroendocrine carcinoma, small cell type with sheets, ribbons, and clusters of small to medium-sized cells with minimal cytoplasm; hyperchromatic, indistinct nucleoli; nuclear molding; and frequent mitotic figures. (E) Low-power sinonasal undifferentiated carcinoma with lobules of pleomorphic cells and lack of squamous/glandular differentiation. (F) Sinonasal undifferentiated carcinoma high-power magnification of large, round cells with prominent nucleoli and varying amount of cytoplasm.

Xp21.1 (in 66\% of cases). Gains at 5q35, 13q, and 20q and losses at $2 \mathrm{q} 31.1,2 \mathrm{q} 33.3$, and $6 \mathrm{q} 16-\mathrm{q} 22$ were present in $50 \%$ of cases. The identified regions of gene copy number change have been implicated in a variety of tumors, especially carcinomas. In addition, these results indicate that gains in $20 \mathrm{q}$ and $13 \mathrm{q}$ may be important in the progression of this cancer and that these regions possibly harbor genes with functional relevance in ENG.

The most common site of origin is in the upper nasal cavity in the region of the cribriform plate. ${ }^{1,2}$ Included in the areas of the proposed origin are Jacobson's organ (vomeronasal organ), sphenopalatine (pterygoid palatine) ganglion, olfactory placode, and the ganglion of loci (nervus terminalis). "Ectopic" origin in the lower nasal cavity or within one of the paranasal sinuses (e.g., maxillary sinus) may occur. ${ }^{1,2}$ ENBs may on occasion present as an intracranial (frontal lobe) mass with involvement of the superior aspect of the cribriform plate or, rarely, may occur intracranially with no intranasal component. ${ }^{9}$

Patients presenting with ENB are typically reported to have a slight male predominance and a bimodal age distribution, with one peak in the second decade of life and the second peak in the sixth decade of life. ${ }^{10}$ However, a recent report from our institution of 70 patients treated from 1992 to 2006, the median age at diagnosis was 51 years with a range 9 to 78 years. ${ }^{11}$ The most common age group of presentation was the sixth decade (20\%), followed by the fifth and seventh decades of life. This observation is confirmed by Jethanamest et al in their population-based analysis of the SEERS database, where patients in the second decade of life comprised fewer than $10 \%$ of the cohort. ${ }^{12}$

Clinically, patients often present late in their disease course and the majority will have Kadish stage $\mathrm{C}$ disease. ${ }^{13}$ 
ENB can grow insidiously, and some studies have reported a delay of 6 to 12 months between onset of symptoms and diagnosis. ${ }^{14,15}$ The most common presenting symptoms are nasal obstruction, followed by epistaxis, with unilateral symptoms more common than bilateral symptoms. ${ }^{9,16-18}$ Other symptoms may include headaches, facial pain, "sinusitis," anosmia, and change in vision, or the nasal mass may be asymptomatic and found incidentally. Symptoms resulting from intracranial invasion are less common. ${ }^{17,19}$ In its early stages, clinical examination typically reveals a unilateral nasal mass arising from olfactory cleft, medial to the middle turbinate. Advanced-stage tumor may manifest with signs of extensive local invasion of the orbit, palate, facial soft tissue, and skin. ENB does not have a specific radiologic appearance. It is a homogenous soft tissue mass in the nasal vault. On magnetic resonance imaging, it is hypointense on T1-weighted images and isointense or hyperintense on T2-weighted images. Although ENB can have intracranial extensions, the radiologic description of a "dumbbell-shaped" mass extending across the cribriform plate is rarely seen.

Multiple studies have demonstrated that surgery combined with radiation is superior to radiation as definitive therapy. ${ }^{9,12,20-23}$ The traditional approach is the anterior craniofacial resection (ACFR), involving a bifrontal craniotomy combined with a transfacial approach. A meta-analysis of 390 patients treated with open ACFR between 1990 and 2000 reported 5-year disease-free survival (DFS) of $45 \%{ }^{9}$ At MD Anderson Cancer Center, the median overall survival (OS) was 126.3 months (10.5 years) and median disease-specific survival (DSS) was 139 months (11.6 years). ${ }^{11}$

Over the past two decades, endoscopic surgery has become an accepted treatment modality in carefully selected patients. Several small series have reported 3- to 5-year DFS of between 89 and $100 \% .^{24-28}$ In a meta-analysis of 23 publications comparing endoscopic to open surgery, endoscopic surgery was associated with better survival (10-year OS of $90 \%$ compared with $65 \%$ for open resection ). ${ }^{23}$ However, the majority of open surgery patients had Kadish stage C or D disease, and there are more cases of long-term follow-up for the open surgery group. Hence, the use of endoscopic surgery for early stage ENB is supported, but long-term follow up is needed.

The University of Virginia group has reported on a cohort of 50 patients treated with neoadjuvant chemotherapy and radiation followed by surgery. Patients with Kadish stage A or $B$ received preoperative radiation therapy, and patients with Kadish stage $C$ received preoperative sequential chemotherapy and radiation therapy. All patients received open ACFR 4 to 6 weeks after completion of radiation therapy. The 5- and 15 -year DFS was 86.5 and $82.6 \%$, respectively. The localregional recurrence was $24 \%$ and distant recurrence was $10 \%$. The authors commented that they did not experience significantly higher surgical complication rates than other series. $^{18}$

Radiation can be used alone or concurrently with chemotherapy in the postoperative setting for patients at high risk of local-regional recurrence. The incidence of local recurrence has been reported to be between 10 to $30 \%$ and is higher in patients with negative prognostic factors such as high Hyams grading, advanced Kadish stage disease, intracranial extension, and positive resection margins. ${ }^{9,13,29}$ These patients have poorer survival outcomes and warrant adjuvant therapy. At our institution, patients treated with surgery with postoperative radiation therapy have a median DSS of 218.5 months compared with 87.9 months for patients treated with surgery alone. ${ }^{11}$ The usefulness of concurrent or neoadjuvant chemotherapy remains unknown.

At presentation, 5 to $8 \%$ of patients will have neck disease. In a meta-analysis by Dulguerov et al, survival was $29 \%$ for patients with lymph node metastasis at presentation compared with $64 \%$ for patients with N0 disease. ${ }^{9}$ These patients should be offered therapeutic nodal dissection with possible postoperative irradiation. Twenty-five percent of patients with untreated necks will develop nodal metastasis. ${ }^{30}$ There are no reports that prophylactic neck dissection is beneficial, but there are limited data that elective neck irradiation (ENI) to cervical nodal basin is associated with a reduction in relapse rates. ${ }^{31}$ Hence, at our institution, we advocate including the upper neck and retropharyngeal nodes in the radiation field.

Despite promising 5-year outcomes, the results of longterm follow-up are variable. At our institution, the median time to recurrence of 6.9 years and the incidence of overall recurrence and distant metastasis (DM) was 46 and 15\%, respectively. $^{2}$ Recurrences have been reported beyond 10 years after the initial diagnosis and two published studies confirm this. In Howard et al's cohort of 56 patients treated from 1978 to 2004, the 5-, 10-, and 15-year DFS was 74, 50, and $40 \%$, respectively. ${ }^{32}$ The University of Virginia reported 15- and 20-year DFS of 82.6 and $81.2 \%$, respectively. ${ }^{18,33}$ Patients treated for ENB warrant long-term follow-up.

\section{Neuroendocrine Carcinoma}

Neuroendocrine tumors are very rare in the nasal cavity, paranasal sinuses, or nasopharynx. The recognizable types are typical carcinoid, atypical carcinoid, and small cell carcinoma neuroendocrine type. It is unclear whether large cell NEC that corresponds to the pulmonary counterpart occurs in these sites. There are also rare cases that do not fit these categories, and the diagnostic label "neuroendocrine carcinoma, not otherwise specified."

\section{Carcinoid Tumor and Atypical Carcinoid}

Typical and atypical carcinoids of the nasal cavity and paranasal sinuses are exceedingly rare, possibly because they are underreported or have been included under other nondescriptive categories, such as "neuroendocrine carcinoma." 34,35 They are otherwise similar to carcinoids in other sites (-Fig. 1C).

\section{Small Cell Carcinoma, Neuroendocrine Type}

Small cell carcinoma, neuroendocrine type (SCCNET) is a high-grade carcinoma composed of small to intermediatesized cells resembling those of small cell carcinoma of pulmonary or extrapulmonary origin. Necrosis, large numbers of apoptotic cells, high mitotic rate, and lack of neurofibrillary stroma are microscopic hallmarks of this tumor ( - Fig. 1D). It 
is synonymous with small cell carcinoma, small cell NEC, oat cell carcinoma, poorly differentiated NEC.

SCCNET of the sinonasal tract is a rare tumor with no sex, racial, or geographic predilection and no known association with smoking or radiation. The age range is from 26 to 77 years with a mean of 49 years. SCCNET most commonly arises in the superior or posterior nasal cavity, and often extends into the maxillary or ethmoid sinuses. Primary tumors of the maxillary or ethmoid sinuses without nasal involvement can be seen in approximately $45 \%$ of cases. Secondary involvement of the nasopharynx is present in a minority of patients. Advanced tumors may invade the skull base, orbit, or brain. Rare tumors have shown elevated serum levels of adrenocorticotropic hormone and calcitonin. ${ }^{35}$

SCCNETs are aggressive tumors with a poor prognosis and frequent local recurrence and DM despite multimodal therapy. ${ }^{35}$ Follow-up data have shown a local recurrence rate of $45 \%$ and a DM rate of $35 \%$. Common sites of metastases include cervical lymph nodes, lung, liver, bone marrow, and vertebrae.

\section{Treatment of Neuroendocrine Carcinomas}

NECs, regardless of differentiation or subtype, account for $5 \%$ of sinonasal malignancies. ${ }^{36}$ The median age of patients presenting to our institution was 56 years, with equal sex distribution. ${ }^{37}$ The majority of patients present with $\mathrm{T} 3$ or T4 disease (78\%) and were node negative (82\%). The most common sites of tumor origin were the ethmoid sinus (64\%), the nasal cavity (32\%), and the maxillary sinus (14\%). Like $\mathrm{ENB}$, the most common presenting symptoms were nasal obstruction, epistaxis, and nasal drainage. Changes in visual acuity were reported by $18 \%$ of patients. Facial pain, facial swelling, diplopia, and V2 numbness were uncommon. ${ }^{37}$

The limited number of cases published, difficulties of diagnosis, and heterogeneity of treatment approaches hamper evaluating the ideal treatment strategy. ${ }^{38-40}$ In a recent publication from our institution spanning 1990 to 2004, approximately half of the cohort received surgery as the primary treatment modality and one-third received chemoradiation therapy. ${ }^{37}$ The 5 -year OS, DSS, and DFS were $66.9 \%$, $78.5 \%$, and $43.8 \%$, respectively. The incidence of local, regional, and distant failure was $21 \%, 25 \%$, and $18 \%$, respectively. These results are better than generally reported. ${ }^{38-40}$ Predictors of poor outcomes were patients with foveal or orbital involvement and tumor originating outside of the nasal cavity. A complete response to neoadjuvant chemotherapy correlated with improved survival at 3 years. ${ }^{37}$ Given the high incidence of distant failure and the chemosensitivity of NEC, neoadjuvant chemotherapy followed by either chemoradiation or surgery and postoperative radiation therapy is a promising strategy. In an earlier report from our institution where 8 of 18 patients were treated with neoadjuvant chemotherapy, the OS and local recurrence were 64.2 and $27.4 \%$, respectively. ${ }^{41}$ However, the regional and distant failures were lower than expected $(12.9 \%$ and $12.3 \%$, respectively), perhaps attributable to the higher proportion of patients receiving neoadjuvant chemotherapy.

\section{Sinonasal Undifferentiated Carcinoma}

The original definition for SNUC was reported by Frierson et al as a highly aggressive and clinicopathologically distinctive carcinoma of uncertain histogenesis that typically presents with locally extensive disease. ${ }^{42,43}$ SNUCs are a rare tumor, with fewer than 200 reported cases. SNUCs form nests, lobules, trabeculae, and sheets, in the absence of squamous or glandular differentiation (-Fig. 1E). Severe dysplasia of the overlying surface epithelium has been noted in a few instances. The nuclei are medium-sized to large, surrounded by small amounts of eosinophilic cytoplasm that lacks a syncytial quality. The nucleoli are variable in size, but most often, they are single and prominent. The mitotic rate is very high and there is often prominent tumor necrosis and apoptosis (-Fig. 1F). The neoplasm is typically negative for Epstein-Barr virus. Some cases have occurred after prior radiation therapy for nasopharyngeal carcinoma. The majority of SNUCs react with simple keratins (CK7, CK8, CK19) and have focal positivity for epithelial membrane antigen, neuron-specific enolase, and p53; synaptophysin and chromogranin may show patchy rare immunoreactivity.

A study looking at cytogenetic alteration in 14 sinonasal carcinomas found that out of five patients with SNUC, two had an abnormal karyotype. ${ }^{44}$ One case of SNUC had a diploid complex karyotype, and the other had a triploid composite with 60 to 69 chromosomes. The chromosome arms that had frequent breakpoints and rearrangements were: $1 \mathrm{p}, 6 \mathrm{p}, 7 \mathrm{p}$, and $12 \mathrm{q} .{ }^{44}$ In a recently established SNUC cell line, by combining G-banding with spectral karyotyping analysis, 12 translocations involving various chromosomes were identified. $^{45}$

SNUCs can present from the third to ninth decade, with a median age in the sixth decade. ${ }^{46}$ There is a slight male predominance. ${ }^{46-48}$ The majority of SNUCs arise in the ethmoid and maxillary sinus. ${ }^{48}$ Epistaxis, nasal obstruction, headache, or facial pain are frequent complaints. Up to $50 \%$ of patients complain of visual acuity changes. ${ }^{48}$ Seventy to $90 \%$ of patients present with stage T4 disease. Up to $50 \%$ present with orbital involvement or skull base and brain involvement. In contrast to the frequent presentation of locally advanced disease, 5 to $13 \%$ of patients have nodal metastasis at presentation. ${ }^{41,46-49}$

Despite earlier reports of very poor outcomes, ${ }^{42,43}$ recent larger series have shown 5-year OS between 40 and 75\%. ${ }^{47-50}$ Treatment strategies vary between institutions, and no single approach has demonstrated a clear therapeutic advantage. In Tanzler et al's series of 15 patients, 9 were treated with open craniofacial resection and postoperative radiation or chemoradiation therapy. The 3-year OS was $67 \%{ }^{49}$ MD Anderson Cancer Center reported an OS of 63\% in their series of 18 patients, equally divided between neoadjuvant chemotherapy as the primary treatment modality in half of the cohort and surgery in the remainder. ${ }^{41} \mathrm{Al}$-Mamgani et al published a series of 21 patients divided between chemoradiation therapy, neoadjuvant chemotherapy, or surgery as primary mode of treatment. Patients with resectable disease were selected for surgery with postoperative radiation or chemoradiation therapy. ${ }^{48}$ Those with unresectable disease were offered 
either neoadjuvant chemotherapy or definitive chemoradiation therapy. Predictors of local control on multivariate analysis were $\mathrm{T}$ staging and treatment with three treatment modalities compared with two modalities. Interestingly, this series reported the best survival outcomes published to date (OS of 74\%), suggesting that a tailored treatment approach is better than any one strategy.

Open craniofacial resection followed by postoperative radiation therapy has been the standard treatment approach. More recently, a small series of patients treated with endoscopic endonasal surgery reported equivalent results in selected patients. ${ }^{51}$ For resectable disease, surgery followed by postoperative radiation therapy results in local-regional control of up to $78 \%{ }^{49}$ Although primary chemoradiation is often reserved for patients with unresectable disease, a metaanalysis by Reiersen et al showed that in patients with the most extensive disease category (Kadish stage C), those treated with surgery and one adjuvant therapy showed improved survival and local-regional control when compared with patients who did not receive surgery ${ }^{46}$ Thus, surgery as primary mode of treatment is acceptable for patients with resectable disease and improves oncologic control when utilized as part of multimodality treatment for unresectable disease.

In the search for better outcomes, chemoradiation therapy as primary treatment modality has been proposed. The University of Virginia published their series of 15 patients treated with upfront radiation with or without chemotherapy followed by surgical resection. ${ }^{47}$ Their 2 -year OS was $47 \%$. To assess the effectiveness of radiation, the authors examined the surgical specimen of the 10 patients who went on to have ACFR. Three patients were found to have no disease in the specimen. Of the seven patients with disease in the specimen, five resulted in clear resection margins and two patients had positive margins.

Given the propensity of SNUC to present with locally advanced disease, neoadjuvant chemotherapy has been advocated as a strategy for downstaging unresectable tumors before definitive surgery or radiation therapy. Rischin et al reported a 2-year OS of $64 \%$ in a series of 10 patients. ${ }^{52}$ Four of the seven patients treated with neoadjuvant cisplatin or carboplatin and fluorouracil showed objective evidence of response and two patients had stable disease with symptomatic improvement. One patient with progressive disease died shortly after completing radiation therapy, supporting preliminary evidence from our institution that response to chemotherapy is a prognostic indicator and may be used to select patients for definite treatment. With DM reported in the range of 10 to $30 \%,{ }^{47-50}$ an additional benefit of neoadjuvant chemotherapy may be the reduction in DM. It is notable that in Rischin et al's study, none of the patients treated with neoadjuvant chemotherapy followed by concurrent chemoradiation therapy developed DM, whereas both patients treated with surgery and radiation therapy did.

Regional failure varies between 10 and $30 \%$ and up to $50 \%$ if the cervical nodal basin is not treated..$^{52} \mathrm{Al}$-Mamgani et al treated $42 \%$ of patients at high risk of regional metastasis with ENI, defined as T4 disease involving skin of cheek, infratemporal fossa, pterygoid plate, or cribriform plate. They reported regional control of 100 and $80 \%$, respectively, for patients who received ENI and those who did not. ${ }^{48}$ This is consistent with other reports published in the literature and supports ENI in patients with locally advanced disease. ${ }^{49,50}$

Patients with early stage disease confined to the nasal cavity may be treated with surgery and postoperative radiation therapy or radiation therapy alone. However, the optimal treatment for patients with advanced local disease remains debated. Regardless of the primary treatment modality selected, multimodality treatment improves survival outcomes. ${ }^{46,48} \mathrm{ENI}$ is recommended for high-risk patients, and the role of neoadjuvant chemotherapy needs to be further studied.

\section{Final Comments}

There are significant differences in prognosis and treatment for ENB, SNUC, and NEC. Most authors agree that patients with ENB may be treated with local therapy alone (surgery and radiation). ENB is characterized by slow-onset symptoms and excellent 5-year survival but with a propensity for delayed local-regional recurrence that warrants long-term follow up. In contrast, patients with SNUC and NEC tend to present with rapid-onset symptoms, advanced local disease, and earlier onset of recurrence. SNUC and NEC are aggressive tumors requiring multimodality treatment that includes chemotherapy. Although the optimal strategy is yet to be determined, individualized treatment that takes into account the stage of tumor, patient comorbidities, and histologic characteristics can achieve better survival. Recent advances such as endoscopic endonasal surgery, neoadjuvant chemotherapy, and proton beam radiation therapy have the potential to improve oncologic outcomes but further investigation in needed.

\section{References}

1 Faragalla H, Weinreb I. Olfactory neuroblastoma: a review and update. Adv Anat Pathol 2009;16(5):322-331

2 Ow TJ, Bell D, Kupferman ME, Demonte F, Hanna EY. Esthesioneuroblastoma. Neurosurg Clin N Am 2013;24(1):51-65

3 Bell D, Saade R, Roberts D, et al. Prognostic utility of Hyams histological grading and Kadish-Morita staging systems for esthesioneuroblastoma outcomes. Head Neck Pathol 2014; May 8 (Epub ahead of print)

4 Holland H, Koschny R, Krupp W, et al. Comprehensive cytogenetic characterization of an esthesioneuroblastoma. Cancer Genet $\mathrm{Cy}$ togenet 2007;173(2):89-96

5 Bockmühl U, You X, Pacyna-Gengelbach M, Arps H, Draf W, Petersen I. CGH pattern of esthesioneuroblastoma and their metastases. Brain Pathol 2004;14(2):158-163

6 Riazimand SH, Brieger J, Jacob R, Welkoborsky HJ, Mann WJ. Analysis of cytogenetic aberrations in esthesioneuroblastomas by comparative genomic hybridization. Cancer Genet Cytogenet 2002;136(1):53-57

7 Szymas J, Wolf G, Kowalczyk D, Nowak S, Petersen I. Olfactory neuroblastoma: detection of genomic imbalances by comparative 
genomic hybridization. Acta Neurochir (Wien) 1997;139(9): 839-844

8 Guled M, Myllykangas S, Frierson HF Jr, Mills SE, Knuutila S, Stelow EB. Array comparative genomic hybridization analysis of olfactory neuroblastoma. Mod Pathol 2008;21(6):770-778

9 Dulguerov P, Allal AS, Calcaterra TC. Esthesioneuroblastoma: a meta-analysis and review. Lancet Oncol 2001;2(11):683-690

10 Klepin HD, McMullen KP, Lesser GJ. Esthesioneuroblastoma. Curr Treat Options Oncol 2005;6(6):509-518

11 Ow TJ, Hanna EY, Roberts DB, et al. Optimization of long-term outcomes for patients with esthesioneuroblastoma. Head Neck 2014;36(4):524-530

12 Jethanamest D, Morris LG, Sikora AG, Kutler DI. Esthesioneuroblastoma: a population-based analysis of survival and prognostic factors. Arch Otolaryngol Head Neck Surg 2007;133(3):276-280

13 Patel SG, Singh B, Stambuk HE, et al. Craniofacial surgery for esthesioneuroblastoma: report of an international collaborative study. J Neurol Surg B Skull Base 2012;73(3):208-220

14 Dulguerov P, Calcaterra T. Esthesioneuroblastoma: the UCLA experience 1970-1990. Laryngoscope 1992;102(8):843-849

15 Zafereo ME, Fakhri S, Prayson R, et al. Esthesioneuroblastoma: 25year experience at a single institution. Otolaryngol Head Neck Surg 2008;138(4):452-458

16 Bachar G, Goldstein DP, Shah M, et al. Esthesioneuroblastoma: The Princess Margaret Hospital experience. Head Neck 2008;30(12): 1607-1614

17 Broich G, Pagliari A, Ottaviani F. Esthesioneuroblastoma: a general review of the cases published since the discovery of the tumour in 1924. Anticancer Res 1997;17(4A):2683-2706

18 Loy AH, Reibel JF, Read PW, et al. Esthesioneuroblastoma: continued follow-up of a single institution's experience. Arch Otolaryngol Head Neck Surg 2006;132(2):134-138

19 Ward PD, Heth JA, Thompson BG, Marentette LJ. Esthesioneuroblastoma: results and outcomes of a single institution's experience. Skull Base 2009;19(2):133-140

20 Gruber G, Laedrach K, Baumert B, Caversaccio M, Raveh J, Greiner R. Esthesioneuroblastoma: irradiation alone and surgery alone are not enough. Int J Radiat Oncol Biol Phys 2002;54(2):486-491

21 Chao KS, Kaplan C, Simpson JR, et al. Esthesioneuroblastoma: the impact of treatment modality. Head Neck 2001;23(9):749-757

22 Ozsahin M, Gruber G, Olszyk O, et al. Outcome and prognostic factors in olfactory neuroblastoma: a rare cancer network study. Int J Radiat Oncol Biol Phys 2010;78(4):992-997

23 Devaiah AK, Andreoli MT. Treatment of esthesioneuroblastoma: a 16-year meta-analysis of 361 patients. Laryngoscope 2009; 119(7):1412-1416

24 Unger F, Haselsberger K, Walch C, Stammberger H, Papaefthymiou G. Combined endoscopic surgery and radiosurgery as treatment modality for olfactory neuroblastoma (esthesioneuroblastoma). Acta Neurochir (Wien) 2005;147(6):595-601, discussion 601602

25 Castelnuovo PG, Delù G, Sberze F, et al. Esthesioneuroblastoma: endonasal endoscopic treatment. Skull Base 2006;16(1):25-30

26 Lund V, Howard DJ, Wei WI. Endoscopic resection of malignant tumors of the nose and sinuses. Am J Rhinol 2007;21(1): 89-94

27 Nicolai P, Battaglia P, Bignami M, et al. Endoscopic surgery for malignant tumors of the sinonasal tract and adjacent skull base: a 10-year experience. Am J Rhinol 2008;22(3):308-316

28 Folbe A, Herzallah I, Duvvuri U, et al. Endoscopic endonasal resection of esthesioneuroblastoma: a multicenter study. Am J Rhinol Allergy 2009;23(1):91-94

29 Monroe AT, Hinerman RW, Amdur RJ, Morris CG, Mendenhall WM. Radiation therapy for esthesioneuroblastoma: rationale for elective neck irradiation. Head Neck 2003;25(7):529-534

30 Zanation AM, Ferlito A, Rinaldo A, et al. When, how and why to treat the neck in patients with esthesioneuroblastoma: a review. Eur Arch Otorhinolaryngol 2010;267(11):1667-1671
31 Noh OK, Lee SW, Yoon SM, et al. Radiotherapy for esthesioneuroblastoma: is elective nodal irradiation warranted in the multimodality treatment approach? Int J Radiat Oncol Biol Phys 2011; 79(2):443-449

32 Howard DJ, Lund VJ, Wei WI. Craniofacial resection for tumors of the nasal cavity and paranasal sinuses: a 25 -year experience. Head Neck 2006;28(10):867-873

33 Levine PA. Would Dr. Ogura approve of endoscopic resection of esthesioneuroblastomas? An analysis of endoscopic resection data versus that of craniofacial resection. Laryngoscope 2009; 119(1):3-7

34 Mills SE. Neuroendocrine tumors of the head and neck: a selected review with emphasis on terminology. Endocr Pathol 1996;7(4): 329-343

35 Mills SE. Neuroectodermal neoplasms of the head and neck with emphasis on neuroendocrine carcinomas. Mod Pathol 2002;15(3): 264-278

36 Renner G. Small cell carcinoma of the head and neck: a review. Semin Oncol 2007;34(1):3-14

37 Mitchell EH, Diaz A, Yilmaz T, et al. Multimodality treatment for sinonasal neuroendocrine carcinoma. Head Neck 2012;34(10): 1372-1376

38 Perez-Ordonez B, Caruana SM, Huvos AG, Shah JP. Small cell neuroendocrine carcinoma of the nasal cavity and paranasal sinuses. Hum Pathol 1998;29(8):826-832

39 Fitzek MM, Thornton AF, Varvares M, et al. Neuroendocrine tumors of the sinonasal tract. Results of a prospective study incorporating chemotherapy, surgery, and combined proton-photon radiotherapy. Cancer 2002;94(10):2623-2634

40 Babin E, Rouleau V, Vedrine PO, et al. Small cell neuroendocrine carcinoma of the nasal cavity and paranasal sinuses. J Laryngol Otol 2006;120(4):289-297

41 Rosenthal DI, Barker JL Jr, El-Naggar AK, et al. Sinonasal malignancies with neuroendocrine differentiation: patterns of failure according to histologic phenotype. Cancer 2004;101(11): 2567-2573

42 Frierson HF Jr, Mills SE, Fechner RE, Taxy JB, Levine PA. Sinonasal undifferentiated carcinoma. An aggressive neoplasm derived from schneiderian epithelium and distinct from olfactory neuroblastoma. Am J Surg Pathol 1986;10(11):771-779

43 Levine PA, Frierson HF Jr, Stewart FM, Mills SE, Fechner RE, Cantrell RW. Sinonasal undifferentiated carcinoma: a distinctive and highly aggressive neoplasm. Laryngoscope 1987;97(8 Pt 1): 905-908

44 Gil Z, Orr-Urtreger A, Voskoboinik N, et al. Cytogenetic analysis of sinonasal carcinomas. Otolaryngol Head Neck Surg 2006;134(4): 654-660

45 Takahashi Y, Kupferman ME, Bell D, et al. Establishment and characterization of novel cell lines from sinonasal undifferentiated carcinoma. Clin Cancer Res 2012;18(22):6178-6187

46 Reiersen DA, Pahilan ME, Devaiah AK. Meta-analysis of treatment outcomes for sinonasal undifferentiated carcinoma. Otolaryngol Head Neck Surg 2012;147(1):7-14

47 Musy PYR, Reibel JF, Levine PA. Sinonasal undifferentiated carcinoma: the search for a better outcome. Laryngoscope 2002;112(8 Pt 1):1450-1455

48 Al-Mamgani A, van Rooij P, Mehilal R, Tans L, Levendag PC. Combined-modality treatment improved outcome in sinonasal undifferentiated carcinoma: single-institutional experience of 21 patients and review of the literature. Eur Arch Otorhinolaryngol 2013;270(1):293-299

49 Tanzler ED, Morris CG, Orlando CA, Werning JW, Mendenhall WM. Management of sinonasal undifferentiated carcinoma. Head Neck 2008;30(5):595-599

50 Chen AM, Daly ME, El-Sayed I, et al. Patterns of failure after combined-modality approaches incorporating radiotherapy for sinonasal undifferentiated carcinoma of the head and neck. Int J Radiat Oncol Biol Phys 2008;70(2):338-343 
S156 Malignant Sinonasal Tumors Su et al.

51 Revenaugh PC, Seth R, Pavlovich JB, Knott PD, Batra PS. Minimally invasive endoscopic resection of sinonasal undifferentiated carcinoma. Am J Otolaryngol 2011;32(6): 464-469
52 Rischin D, Porceddu S, Peters L, Martin J, Corry J, Weih L. Promising results with chemoradiation in patients with sinonasal undifferentiated carcinoma. Head Neck 2004;26(5): 435-441 